\title{
Spin-Charge Separation In The 1D Hubbard Model With Longer-Range Hop- ping.
}

\section{C.W.M. Castleton and M.W. Long}

School of Physics, University of Birmingham, Edgbaston, Birmingham, B15 2TT, England.

Although it is very hard to solve the 2D Hubbard model in the $U \rightarrow \infty$ limit, progress can be made using a class of $1 \mathrm{D}$ models in which additional longer range-hopping is included. Here, an unusual form of mean field theory is used to study the charge motion in the simplest model of the class - that with nearest and next next nearest neighbour hopping- The solutions found are all spin-charge separated, and for lower electron densities, indications of hard core boson like behaviour for the charge degrees of freedom are seen. For higher electron densities Nagaoka ferromagnetism is seen.

\section{INTRODUCTION}

The authors recently obtained exact results ${ }^{1}$ for a $1 \mathrm{D}$ $U=\infty$ model involving both nearest neighbour $\left(t_{1}\right)$ and next next nearest neighbour hopping $\left(t_{3}\right)$, in the limit that $t_{3} \rightarrow 0$. The spin and charge degrees of freedom separated, and a phase transition was predicted from a quantum Neél state to a Nagaoka ${ }^{2}-l i k e$ ferro magnetic state, above electron density $\mathrm{n}=0.6675045$. In the current work, an unusual form of mean field theory is used to study the model for finite values for $t_{3}$. Similar results are obtained.

\section{EXPRESSING THE MODEL.}

The model we start from can be expressed as

$$
\hat{H}=-t_{1} \sum_{i \sigma}\left[f_{i \sigma}^{\dagger} f_{i+1 \sigma}+c . c\right]-t_{3} \sum_{i \sigma}\left[f_{i \sigma}^{\dagger} f_{i+3 \sigma}+c . c\right]
$$

where $f_{i r}, f_{i \sigma}^{\dagger}$ are the normal electron creation and annihilation operators, with double occupancy already projected out. Previously, ${ }^{1}$ an exact mapping was derived to a basis in which the spin and charge degrees of freedom are described separately

$$
f_{i_{1} \sigma_{1}}^{\dagger} f_{i_{2} \sigma_{2}}^{\dagger} \ldots . f_{i_{n} \sigma_{n}}^{\dagger}|0\rangle \rightarrow c_{i_{1}}^{\dagger} c_{i_{2}}^{\dagger} \ldots c_{i_{n}}^{\dagger}\left|\sigma_{1} \sigma_{2} \ldots \sigma_{n}\right\rangle
$$

$c_{i^{\prime}}, c_{i}^{\dagger}$ create and annihilate fermionic charges. The spins are controlled by "ring exchange" operators $\hat{R}_{\alpha, \alpha \alpha^{\prime}}$, which cyclically permute the spins between $\alpha$ and $\alpha^{\prime}$, as numbered along a "compressed spin chain", ie the spins carry the labels, not the sites. As shown in the previous papers, equation (1) can be reexpressed exactly in terms of the new basis, using terms involving $c_{i}, c_{i}^{\dagger}$ only, and other terms involving $c_{i}$, $c_{i}^{\dagger}$ with $\hat{R}_{\alpha, \alpha^{\prime}}$.

\section{THE MEAN FIELD SOLUTION.}

The approximation used here involves studying the spins in the mean field of the charges, and the charges in the mean field of the spins. Thus any correlations between the spin excitations and charge excitations are lost.

The resulting charge Hamiltonian is

$$
\begin{aligned}
& \hat{H}_{\text {charge }}=-t_{1} \sum_{i}\left[c_{i}^{\dagger} c_{i+1}+c c\right] \\
& -t_{3} \sum_{i}\left[c_{i}^{\dagger} c_{i+3}\left(1-c_{i+1}^{\dagger} c_{i+1}\right)\left(1-c_{i+2}^{\dagger} c_{i+2}\right)+c . c\right] \\
& -t_{3} R_{1} \sum_{i}\left[c_{i}^{\dagger} c_{i+3}\left(\begin{array}{c}
c_{i+1}^{\dagger} c_{i+1}\left(1-c_{i+2}^{\dagger} c_{i+2}\right) \\
+\left(1-c_{i+1}^{\dagger} c_{i+1}\right) c_{i+2}^{\dagger} c_{i+2}
\end{array}\right)+c c\right] \\
& -t_{3} R_{2} \sum_{i}\left[c_{i}^{\dagger} c_{i+3} c_{i+1}^{\dagger} c_{i+1} c_{i+2}^{\dagger} c_{i+2}+c c\right]
\end{aligned}
$$

The three $t_{3}$ terms hop a charge over 0,1 or 2 other charges respectively, hence the appearance of $R_{1}=<$ $\hat{R}_{\alpha, \alpha+1}>$ in the second, from two spin ring exchanges, and $R_{2}=<\hat{R}_{\alpha, \alpha+2}>$ in the third, from three spin ring exchanges.

The spin Hamiltonian is more easily written in terms of the more traditional $\hat{S}_{\alpha}$ operators -

$$
\hat{H}_{\text {spin }}=C+\frac{J_{1}}{2} \sum_{\alpha} \hat{S}_{\alpha \cdot} \hat{S}_{\alpha+1}+\frac{J_{2}}{2} \sum_{\alpha} \hat{S}_{\alpha} \cdot \hat{S}_{\alpha+2}
$$


where $C, J_{1}$ and $J_{2}$ are charge correlation functions. For $t_{3} \rightarrow 0$, we found $J_{2}<0$ and $J_{1}$ of either sign. Thus spin states similar to the Heisenberg ground state, quantum Neél, and ferromagnet might be expected. These three were chosen as trial spin states to provide values of $R_{1}$ and $R_{2}$ to insert into the charge Hamiltonian, (3).

In addition to this, if the $t_{1} / t_{3}$ model for hard core bosons is expressed in terms of fermionic operators using the Jordan-Wigner transformation ${ }^{3}$, the result is equation (3) with $R_{1}=-1$ and $R_{2}=+1$. This allows direct comparison of our charge results with those for hard core bosons.

The solutions to the charge system were found using a form of Hartree-Fock approximation in which pairing correlations $\left(c_{i}^{\dagger} c_{i+1}^{\dagger}, c_{i+1} c_{i}\right.$, etc $)$ are also permitted. This leads to BCS-like equations, then to a set of integral equations. These are solved self consistently.

\section{THE RESULTS.}

Graph 1 shows the energies of the charge ground states subject to the spin wavefunctions described above, versus $\mathrm{n}$, with $t_{1}=1.0$ and $t_{3}=0.5$. The Heisenberg groundstate does best for $\mathrm{n} \sim<0.6$,

\section{Graph 1.}

Energies for $t_{1}=1$ and $t_{3}=0.5$.

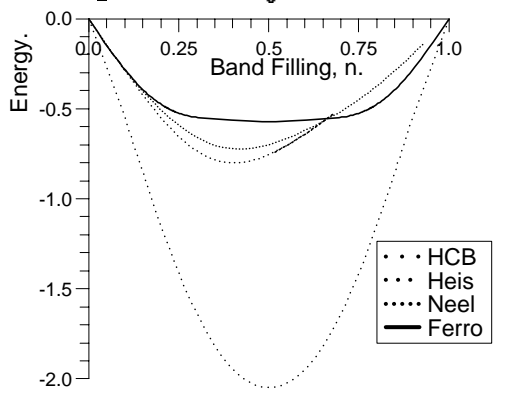

then, briefly, the Neél state, then the solution becomes ferromagnetic. The charge motion for both Heisenberg and Neél spin states is similar to that of hard core bosons. This is illustrated by the pairing correlations in graph 2, which the ferromagnetic state cannot use. Graph 3 shows the $t_{3}$ versus $n$ phase diagram. Qualitatively, the results are like those for the $t_{3} \rightarrow 0$ limit, although the phase transition into the ferromagnet does move to slightly lower $n$ for $t_{3}>\frac{1}{3}$. Study of correlation functions shows that for $t_{3}>\frac{1}{3}$ the charge occupation numbers in the ferromagnetic state are altered to raise the probability of hopping over 0 or 2 other charges, relative to hopping over 1 . This avoids phase cancellation due to Fermi statistics.
Graph 2.

Pairing correlations.

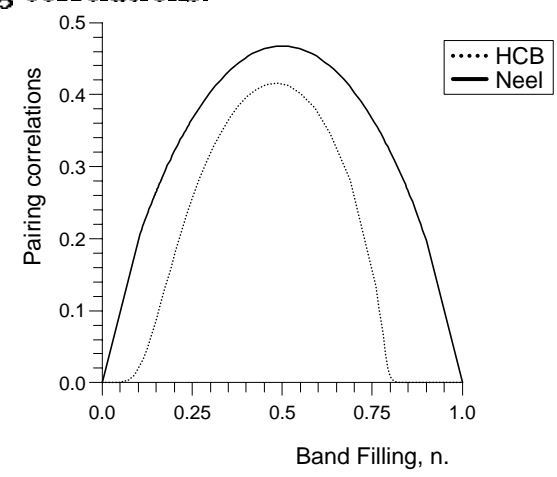

Graph 3.

Electron density $/ t_{3}$ phase diagram.

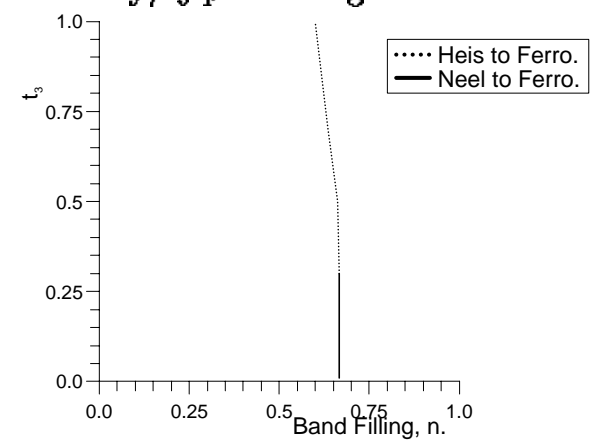

\section{CONCLUSIONS.}

In this mean-field approximation, a transition from a low spin state to Nagaoka ferromagnetism is found at $n \simeq \frac{2}{3}$ for all $t_{3}$, in agreement with the $t_{3} \rightarrow 0$ exact results. For low spin states, the charges use pairing correlations to gain energy from $t_{3}$, becoming similar to hard core bosons. For high spin states with $t_{3}>\frac{1}{3}$, energy is gained by preferentally hopping over even numbers of other charges. The ferromagnet is relatively stabilised by increasing $t_{3}$, but this could well be an artifact of the approximation scheme: for the ferromagnet the exotic spin-charge mean field is exact, whereas for the more complex low spin states it is variational. Hence the latter would gain energy if short range correlations between the spin and charge systems were included.

\section{REFERENCES.}

1. Long, Castleton, Hayward. J. Phys. Condens. Matter. 6481 (1993);6 2757 (1994)

2. Nagaoka. Phys. Rev. 147,392 (1966)

3. Jordan, Wigner. Z. für Phys. 47 ,631 (1928) 